\title{
Realitätsillusion der deutschen Klimapolitik
}

\author{
Deutschland sah sich lange als Vorreiter in der Klimapolitik. Die Bilanz ernüchtert allerdings. \\ Die klimapolitischen Ziele werden zum großen Teil nicht erreicht, und die gesellschaftlichen \\ Kosten der umweltpolitischen Maßnahmen sind sehr hoch. Die Ineffektivität und Ineffizienz der \\ bisherigen Klimapolitik in Deutschland erfordert einen grundlegenden Strategiewechsel, der \\ nur mit einem einheitlichen $\mathrm{CO}_{2}$-Preis für alle Sektoren erreicht werden kann.
}

Der Schutz des Klimas stellt - auch in der aktuell von Corona beherrschten Zeit - eine große Herausforderung dar. Der Klimawandel ist ein globales Phänomen, das alle Menschen weltweit betrifft und zu dem alle Länder einen Beitrag leisten sollten. Im Sinne der Vermeidungsstrategie zielt Klimaschutz daher auf die Reduktion der anthropogenen Treibhausgasemissionen, die vom „Weltklimarat“ (IPCC) als Hauptursache des Klimawandels angesehen werden. Klimaschutz ist ein globales öffentliches Gut, das unter der bekannten „Trittbrettfahrer“-Problematik leidet. Ein einzelnes Land, das seinen Treibhausgasausstoß senkt, trägt zwar die Kosten, die diese Reduktion zur Folge hat (Vermeidungskosten); von der Reduktion profitieren aber weltweit alle Länder, auch wenn sie selbst nicht dazu beitragen. Somit hat jedes Land nur einen geringen Anreiz, selbst in den Klimaschutz zu investieren, wenn gleichzeitig die Vorteile weltweit verteilt werden. Nationalstaatliche Klimapolitik beinhaltet Maßnahmen, die im Inland Wohlfahrtseinbußen verursachen und zum weltweiten Ziel der $\mathrm{CO}_{2}$-Vermeidung nur geringfügig beitragen.

Klimapolitik ist ein internationales Gemeinschaftsgutproblem, es ist kein Informationsproblem und kein Wahrnehmungsproblem. Darauf hat der Wissenschaftliche Beirat beim Bundeswirtschaftsministerium 2016 deutlich hingewiesen. Die Gemeinschaftsgutproblematik lässt sich daher nur mit einer freiwilligen wechselseitigen Kooperation der souveränen Staaten lösen. Daher fordert der Sachverständigenrat zur Begutachtung der gesamtwirtschaftlichen Entwicklung 2019 (SVR) eindringlich, dass die globale Koordination ein wesentliches Element der deutschen Klimapolitik bilden und zu einer weltweit einheitlichen $\mathrm{CO}_{2}$-Bepreisung beitragen sollte. Ein internationaler $\mathrm{CO}_{2}-$ Emissionshandel mit allen Ländern stellt die zielgenaueste

(C) Der/die Autor(en) 2020. Open Access: Dieser Artikel wird unter der Creative Commons Namensnennung 4.0 International Lizenz (https:// creativecommons.org/licenses/by/4.0/deed.de) veröffentlicht.

Open Access wird durch die ZBW - Leibniz-Informationszentrum Wirtschaft gefördert.

* Der Autor gibt seine persönliche Meinung wieder. und kosteneffizienteste Emissionsreduktion sicher. Dies ist aber genau das Gegenteil von dem, wofür das Pariser Klimaschutzabkommen 2015 mit seinen unkoordinierten, unverbindlichen nationalen $\mathrm{CO}_{2}$-Minderungszusagen steht (Weimann, 2016). Auf der UN-Klimakonferenz im Dezember 2015 in Paris hatten sich 197 Staaten verpflichtet, die Erderwärmung bis 2050 auf deutlich unter $2^{\circ} \mathrm{C}$, möglichst sogar unter $1,5^{\circ} \mathrm{C}$ im Vergleich zum vorindustriellen Niveau zu begrenzen und langfristig klimaneutral zu werden. Alle 197 Länder haben sich freiwillig verpflichtet, durch geeignete nationale Maßnahmen zum gemeinsamen Ziel der Begrenzung der Erderwärmung beizutragen.

Die Klimapolitik in Deutschland wird vom ErneuerbarenEnergien-Gesetz (EEG) gestaltet und ist - nach Meinung des SVR und des Wissenschaftlichen Beirats - kleinteilig, teuer und ineffizient und leistet (fast) keinen Beitrag zur klimapolitisch erwünschten Verringerung der Treibhausgas-Emissionen (THG). Der Kohleausstiegsbeschluss und das „Klimapaket 2030" sind weitere Weichenstellungen der deutschen Klimapolitik, die diese Linie fortführen. Aber warum ist die Klimapolitik in Deutschland so unbefriedigend ineffizient, teuer und ineffektiv? Warum unterliegen wir in Deutschland einer Realitätsillusion in der Klimapolitik und wollen sie uns weiterhin schönreden? Und was steht dem vom SVR (2019) geforderten klimapolitischen Neuaufbruch entgegen?

Die Gründe dafür erscheinen vielfältig: Nach Ansicht vieler scheint die Politik nicht ausreichend lernwillig oder nicht lernfähig zu sein und kann sich falsche Entscheidungen nur unzureichend eingestehen. Sie verheddert sich zu sehr in Verteilungskämpfen mit EEG-Lobbygruppen

Dr. Rupert Pritzl ist Mitarbeiter im Bayerischen Staatsministerium für Wirtschaft, Landesentwicklung und Energie in München. 
und inszeniert letztlich nur vorgebliches Bemühen. Politiker neigen eher zu planwirtschaftlichen als zu marktwirtschaftlichen Instrumenten und geben leicht dem Drang nach verteilungspolitischen Markteingriffen nach. Diese Gründe werden im Folgenden aus Sicht der Neuen Institutionenökonomik analysiert (Erlei et al., 2016, $321 \mathrm{ff}$.$) .$

\section{Pfadabhängigkeit des politischen Handelns}

Die Klimapolitik wird in Deutschland maßgeblich vom EEG gestaltet, das den Ausbau der erneuerbaren Energien und insbesondere die Förderung der regenerativen Stromerzeugung vorsieht (Fell, 2020, 19). Am 1. April 2000 trat das EEG in Kraft, das vom damaligen Bundesumweltminister Jürgen Trittin vorangebracht wurde. Es löste das Stromeinspeisegesetz aus dem Jahr 1991 ab und garantiert feste Einspeisevergütungen, die Anschlusspflicht für erneuerbare Energieanlagen und den Einspeisevorrang für Strom aus erneuerbaren Energien. Kernelement ist die technologiespezifische umlagefinanzierte Förderung von erneuerbare Energien über eine garantierte Laufzeit von 20 Jahren (sogenannte EEG-Umlage).

In Deutschland hat sich in den vergangenen Jahren ein historisch gewachsener Wildwuchs unterschiedlicher Steuern, Abgaben und Umlagen auf verschiedene Formen des Energieverbrauchs herausgebildet, der einer effektiven und kosteneffizienten Klimapolitik im Weg stehen (WB, 2019, 4). Dieser Wildwuchs verzerrt die Investitionsentscheidungen von Haushalten und Unternehmen, führt zu hohen zusätzlichen Kosten und erbringt (fast) keine Fortschritte bei der Verringerung der $\mathrm{CO}_{2}$-Emissionen (Frondel, 2017, 14 f.; Bardt und Schaefer, 2018).

Der Wissenschaftliche Beirat hatte schon 2004 darauf hingewiesen, dass eine direkte Förderung erneuerbarer Energien (durch das EEG) innerhalb des übergreifenden EU-Emissionshandelssystems keinen Beitrag zur Verringerung der THG-Emissionen erbringt (WB, 2004, 6). Und viele Experten fragen sich, wie man mit regenerativen Energien und - auf absehbare Zeit - ohne ausreichende Stromspeicher die Versorgungssicherheit für den Industriestandort Deutschland in Zukunft garantieren möchte. Diese grundlegende Sachlogik aber hat wenig Eingang in den politischen Diskurs und in die Entscheidungen der politisch Verantwortlichen gefunden. Ebenso wenig die ernüchternde Bilanz der deutschen Klimapolitik, nach der die $\mathrm{CO}_{2}$-Emissionen in Deutschland in den vergangenen zehn Jahren fast unverändert geblieben sind (Dubbers et al., 2019). Auch die hohen Kosten und Ineffizienzen des EEG (so betragen die spezifischen $\mathrm{CO}_{2}$-Vermeidungskosten der Windenergie onshore rund 1.900 Euro und der Photovoltaik rund 1.874 Euro - gegenüber dem derzeitigen Marktpreis im EU-ETS von rund 28 Euro/t $\mathrm{CO}_{2}\left(\mathrm{CO}_{2}-\right.$
Abgabe 2019, 10)) haben scheinbar kaum zur Einsicht der politisch Verantwortlichen und zu keinem grundlegenden Umsteuern in der Klimapolitik geführt. Im Gegenteil: Weite Teile der Bevölkerung halten nach wie vor am positiv besetzten Bild von den „Erfolgen“ der Energiewende fest und verklären weiterhin die klima- und energiepolitische Fehlsteuerung der Energiewende (Kemfert, 2019).

Aus der Politikberatung ist bekannt, dass Politiker bestrebt sind, eine möglichst klare politische Haltung in bestimmten Politikbereichen einzunehmen, um sich so von Vertretern anderer politischer Parteien abzugrenzen. Diese politischinhaltliche Positionierungen können dann nicht so leicht von Wissenschaftlern oder Interessenvertretern mit einer wissensbasierten Aufklärungs- oder Informationsoffensive verändert werden. Veränderungen in der nach außen verkündeten politischen Positionierung von Abgeordneten sind entweder gar nicht, nur marginal oder nur auf längere Sicht möglich. Politiker fühlen sich auch an die Wert- und Richtungsentscheidungen ihrer Partei gebunden, die wiederum nur schrittweise und durch Parteitagsbeschlüsse in bestimmten zeitlichen Abständen geändert werden können. Parteien sind dabei darauf bedacht, keine abrupten Kurswechsel zuzulassen, die möglicherweise ihre politische Geschlossenheit infrage stellt und Zweifel an der Zuverlässigkeit ihrer politischen Beschlüsse aufkommen lässt (Lindner, 2005, 72 f.). Dies schränkt zwangsläufig die Veränderungsbereitschaft der politischen Vertreter ein. Ein Politiker, der sich einmal öffentlich für das EEG positioniert hat, wird sich dann kaum von Argumenten hinsichtlich der Ineffizienz und Ineffektivität des EEG überzeugen lassen. So wird verständlich, warum das EEG auch nach 20 Jahren Bestand hat und nicht grundlegend verändert wurde. Das EEG hat enorme Beharrungstendenzen.

\section{Funktionsweise des politischen \\ Entscheidungsprozesses}

Das EEG hat eine umfangreiche kleinteilige Regelungsdichte im Energiebereich hervorgebracht, die sich gegen grundlegende Reformmaßnahmen und Abbau der Privilegien wehren konnte. Verschiedene durchsetzungsstarke Interessengruppen bemühen sich - weitgehend erfolgreich - um diese EEG-Einnahmen und eine finanzielle Privilegierung, an der Wiederwahl orientierte politische Entscheidungsträger gewähren zahlreiche finanzielle Vorteile und Sondervorteile (z. B. über Ausnahmetatbestände im EEG) und die nur schwer organisierbare große Gruppe der Stromverbraucher trägt letztlich die Gesamtkosten (Cassel 2017, 46 ff.). Starke Lobbyakteure sind die Erneuerbare-Energien-Verbände (Wind- und Solarlobby), die Unternehmen der Energiewirtschaft und die einzelnen Bundesländer, die sich für ihre natürlichen Standortvorteile (z. B. Wind im Norden, Sonne im Süden und Bioenergie in den agrarischen Flächenstaaten) 
stark machen. Auch die Klimaschutzbürokratie (in Deutschland: das Umweltbundesamt) entwickelt eine Eigendynamik und nutzt ihre politische Stellung - in der Bürokratietheorie William Niskanens - zur Durchsetzung ihrer politischen Präferenzen (Schnellenbach, 2020).

Die durch das EEG gewährten Renteneinahmen haben zu einer Herausbildung mächtiger Interessengruppen beigetragen und zu einem Rent-Seeking-Bereich par excellence geführt (Ehler, 2019, 43 ff.). Die Lobbyakteure streben den weiteren mengenmäßigen Zubau von ErneuerbarenEnergie-Anlagen an, der ihren Mitgliedern lukrative EEGVergütungen für 20 Jahre garantiert (Quasi-Renten), und wehren sich gegen eine Verringerung der Vergütungssätze bzw. Verschlechterung der Einnahmensituation. Die Kosten werden vor allem über die EEG-Umlage, die 2020 auf den Rekordstand von 6,765 ct/KWh angestiegen ist, auf die Stromverbraucher abgewälzt. Die Gesamtkosten von EEG-Umlage, Stromsteuer und Netzentgelte belaufen sich mittlerweile auf rund 35 Mrd. Euro pro Jahr, was durchschnittlich jährlich jeden Bundesbürger mit 426 Euro belastet (der Bundesrechnungshof beziffert das Volumen für 2017 auf 34 Mrd. Euro $(2018,3))$. Das EEG führt zudem zu gesellschaftlich unerwünschten Umverteilungen zugunsten von Eigenheim- und Flächenbesitzern sowie Kapitaleignern, die zulasten der einkommensschwächeren Haushalte und Mieterhaushalte gehen (Techert et al., 2012).

Es kennzeichnet den enormen Machteinfluss der EEGLobbygruppen, dass trotz früh geäußerter Kritik (WB, 2004, 6 ff.) sich an der Grundstruktur dieser kleinteiligen, ineffizienten und sozial unausgewogenen Förderung wenig geändert hat. Je mehr die ökonomische Bedeutung dieser Branche zunimmt und je größer der Anteil der Bevölkerung wird, der selbst EE-Anlagen betreibt, desto weniger könnte die Politik in Zukunft willig bzw. fähig sein, gegen deren Interessen als homogene und gut organisierbare Wählergruppe zu handeln und eine erforderliche vom Sachverständigenrat geforderte Neuausrichtung der Energie- und Klimapolitik vorzunehmen. So manche Vertreter der EEG-Lobby wittern in den aktuellen CoronaZeiten Morgenluft und plädieren für eine Weiterentwicklung der EEG-Förderung, indem sie einen beschleunigten Ausbau von Photovoltaik und Windkraft mit einem expansiven Investitions- und Konjunkturprogramm verknüpfen.

\section{Fehlendes Vertrauen der Politiker in marktliche Prozesse}

Wirksamer Klimaschutz erfordert eine drastische Verringerung der globalen Treibhausgasemissionen und damit eine umfassende Transformation der Energieversorgungssysteme und eine grundlegende Umstrukturierung des gesamten Wirtschaftens und Konsumierens im Hin- blick auf Treibhausgasneutralität. Da diese wirtschaftlichen Umstrukturierungsprozesse enorm kostenintensiv sind, kommt der Kosteneffizienz der klimapolitischen Maßnahmen zentrale Bedeutung zu (Weimann und Timme, 2019). Bestmögliche Kosteneffizienz kann aber nur die marktwirtschaftliche Koordination bewirken, die mit dezentralen Preissignalen dafür sorgt, dass Klimabelastungen dort vermieden werden, wo die Kosten der Vermeidung am niedrigsten sind. Nur der Wettbewerb bildet nach von Hayek den gesellschaftlichen Suchprozess für die ökonomisch effizientesten Lösungen. Die Monopolkommission $(2009,13)$ weist darauf hin, dass die ökonomischen Ineffizienzen eine Volkswirtschaft belasten und damit den Handlungsspielraum für die Politik zur Erreichung weiterer Klimaziele verringern. Die privaten, im Wettbewerb miteinander stehenden Unternehmen sind dabei die wichtigsten Akteure und sollten als Verbündete im Klimaschutz gesehen werden (Göbel, 2019, 26 ff.).

Politische Entscheidungsträger haben ein starkes Interesse, nicht nur den Ordnungsrahmen für die Wirtschaft zu gestalten, sondern im wirtschaftlichen Geschehen bei Einzelentscheidungen selbst mitzuwirken. Politiker wollen unmittelbar an möglichst vielen konkreten (auch wirtschaftlichen) Entscheidungen so beteiligt sein, dass ihnen durch ihr Handeln ein positives Ergebnis zugerechnet wird. Sie wollen auch im wirtschaftlichen Leben sichtbar und wahrnehmbar sein. Sie haben im Regelfall nur geringes Interesse, einen unpersönlichen und ergebnisoffenen Marktmechanismus zu etablieren und ihm bestimmte Entscheidungen anzuvertrauen. Es liegt gar nicht in der Handlungslogik von Politikern, öffentlichkeitsbedeutsame Fragen in den ökonomischen Bereich auszulagern und den sich auf dem Markt bildenden Preisen eine Chance zu geben (Schnellenbach, 2020, 39).

Die politische Entscheidungslogik ist auf direkte, kurzfristig wirkende und zurechenbare Eingriffshandlungen der Politiker ausgerichtet. Wenn ein Marktergebnis aus politischer Sicht als unerwünscht interpretiert wird, wird es schnell als „Marktversagen“ abgewertet und eine politische Intervention gefordert. In vielen Fällen macht diese Intervention dann aber wiederum weitere politische Regulierungen erforderlich, sodass es zu einer Interventionsspirale mit immer weiteren legislativ-administrativen Eingriffen kommt. Renate Köcher $(2020,6)$ beschreibt diese politische Interventionsdynamik kurz und prägnant: „Regulierung macht offensichtlich Lust auf mehr.“ Nur in seltenen Fällen werden marktverzerrende staatliche Eingriffe zurückgenommen und Bürokratie oder legislative Anforderungen abgebaut. Das EEG ist ein gutes Beispiel dafür, dass sich in den vergangenen 20 Jahren ein ausdifferenzierter und selbst von Fachleuten kaum mehr „handhabbares bürokratisches Monster“ (Fell, 2020) herausge- 
bildet hat, das sich allein vom Umfang von einst zwölf auf inzwischen 79 Paragrafen versechsfacht hat. Das Standardwerk „Energierecht" bringt es mittlerweile auf fast 1.800 Seiten (gegenüber 512 Seiten der 1. Auflage 2000).

\section{Ideologie und Gerechtigkeitsvorstellung}

In der gesellschaftlichen Debatte ist immer stärker der Ruf nach staatlich motivierten Eingriffen zu vernehmen. Immer häufiger kommt es dazu, dass der Staat die Marktergebnisse aus politischer Sicht bewertet und dann die Preise in eine von inm gewünschte Richtung beeinflusst (Bernau, 2020, 20). Mit direkten staatlichen Preiseingriffen können Politiker bestimmten Bevölkerungs- und Wählergruppen finanzielle Vorteile zukommen lassen, ohne dass es den Staat etwas kostet. Die gesellschaftlichen Kosten der Preiseingriffe werden auf andere Bevölkerungsgruppen (bei staatlichen Höchstpreisen - auf die Anbieter und Produzenten) abgewälzt.

Viele Ökonomen beklagen heutzutage, dass die Marktwirtschaft immer häufiger ideologisch abgelehnt oder marktliche Prozesse gar nicht zugelassen werden. Der Staat wird von vielen als Wirtschaftslenker und Preissetzer (miss-)verstanden, der alles und für alle regeln soll. Dies ist eine grundlegende Delegation der Entscheidung und der gesellschaftlichen Verantwortlichkeit weg von den Individuen hin zum Staat. Clemens Fuest (2020) spricht hier von einer "schleichenden Verbreitung des Neodirigismus“.

Ist nicht die Bundesumweltministerin Svenja Schulze der Ansicht, dass ,jeder Sektor seinen Teil zur Verringerung der $\mathrm{CO}_{2}$-Emissionen beitragen soll" eine Ablehnung des marktwirtschaftlichen Koordinationsprinzips in der deutschen Klimapolitik? Mit diesen Forderungen ist unterschwellig meist eine offene oder versteckt vorgetragene Wachstums- und Kapitalismuskritik verbunden. Klimaschutz sehen viele als einen geeigneten Weg, das aus anderen Gründen schon immer unerwünschte private Unternehmertum und den unliebsamen marktlichen Wettbewerb auszuhebeln (Göbel, 2019, 26). Die Klimathematik wird gezielt als Hebel instrumentalisiert, um politische Regulierungen zu erzwingen, die man sich schon immer von der Politik erhofft hatte (Schnellenbach, 2020, 39).

\section{Vermeidung kognitiver Dissonanzen}

Zwischen dem politischen Versprechen der Klimaneutralität Deutschlands bis 2050 und den bisherigen Erfolgen der Klimapolitik klafft eine enorme Anspruchslücke. Die Politik formuliert in der Klimapolitik zwar ambitionierte Ziele, viele Menschen fragen sich jedoch, wie die Politik diese Erwartungen realistischerweise jemals erfüllen kann. In der Sozialpsychologie wird dieses Phänomen als „kognitive Dissonanz“ (Leon Festinger, 1978) bezeichnet. Danach stehen mehrere Kognitionen (also Erkenntnisse über die Realität) einer Person zueinander im Widerspruch und charakterisieren die innere Spannung bzw. Zerrissenheit des Individuums. Kognitive Dissonanz gefährdet das stabile Selbstkonzept des handelnden Menschen und drängt nach Auflösung dieser inneren Spannung. Diese Auflösung wird aber nicht dadurch erreicht, dass eine Änderung von Einstellung oder vom Verhalten angestrebt und somit eine der beiden Extrempole aufgegeben wird. Das Ziel Klimaneutralität 2050 aufzugeben, würde bedeuten, dass die Glaubwürdigkeit der Klimapolitik der Bundesregierung und mithin der gesamten Klimapolitik nach der Pariser Klimaschutzkonferenz 2015 beschädigt wird. Und hinsichtlich einer realistischen Zielerreichung dämmert der Politik mittlerweile auch, dass sie die geeigneten Instrumente zur Zielerreichung kaum besitzt.

Eine hilfreiche Lösung bietet die Vorstellung einer „Inszenierung des ehrlichen Bemühens" auf möglichst breiter Ebene und mit der größtmöglichen gesellschaftlichen Beteiligung getreu dem Goetheschen Motto: „Wer immer strebend sich bemüht, den können wir erlösen.“ Denn alle Menschen (auch die politischen Entscheidungsträger) möchten (moralisch) gut handeln, sie möchten ein gutes Gewissen haben und - wie Armin Nassehi $(2019,9)$ formuliert - „auf der richtigen Seite stehen". Auf diese Weise versuchen sie, ihre kognitiven Dissonanzen zu reduzieren und sich so ihr stabiles Selbstkonzept zu erhalten. Daher streben Politiker kleine sichtbare Schritte bzw. Fortschritte an, um darauf hinzuweisen, es geht ja - zumindest ein wenig - in die richtige Richtung. In der aktuellen Klima- und Energiepolitik findet man zahlreiche Belege für diese politische Inszenierungsstrategie: Immer mehr quantitative und immer ambitioniertere Ziele, mehr Personal in den einschlägigen Behörden, mehr Gesetze und Verordnungen, zusätzliche Gremien und Abstimmungsrunden (sogenanntes Klimakabinett der Bundesregierung) sowie ein umfassendes jährliches Monitoringwesen und mehr Öffentlichkeitsarbeit und Kampagnen (Kübler, 2020, 41). Auch die starke Fokussierung von Politik und Öffentlichkeit auf den Stromsektor, in dem der Anteil erneuerbarer Energien mittlerweile über $45 \%$ erreicht (im April 2020 sogar: $52 \%$ ), dient als eine Form der politischen Selbstinszenierung, denn vor allem die Photovoltaik-Industrie erfreut sich weitgehend konsensualer medialer und politischer Interessiert- und Beliebtheit.

In diesem Sinne ist auch der stark emotionale und moralisierende Grundton der gesellschaftlichen Klimadebatte in Deutschland zu sehen, mit dem das klimapolitische Engagement gerechtfertigt und einer fachlich-objektiven Überprüfung entzogen werden soll. Die privaten und politischen Akteure profilieren sich in der Öffentlichkeit als „Klimaretter“ und kaschieren erfolgreich ihre privaten 
Interessen und sind sich einig, dass sie mit der bisherigen Politik so fortfahren (Wetzel, 2020). Angesichts einer wenig stringenten Klimapolitik der Bundesregierung wird diese vermeintliche gesellschaftliche Akzeptanz für den weiteren Ausbau der Erneuerbaren Energien willkommen geheißen, die hohen gesellschaftlichen Kosten und Ineffizienzen verschleiert und die regressiven Verteilungswirkungen hingenommen. In der sich wechselseitig bestärkenden Auffassung, ja moralisch gut zu handeln, werden kritische politikberatende Stimmen vom Sachverständigenrat oder Wissenschaftlichen Beirat (SVR, 2019; WB, 2019) oder kritische kontrollierende Stimmen des Bundesrechnungshofes (2018) einfach beiseite gewischt. Mit der fortwährenden Verschärfung der klimapolitischen Ziele demonstrieren Politiker letztlich nur ihre „hyperambitionierte Ambitionslosigkeit“ (Richter, 2020), und müssten doch eigentlich zugeben, dass sie die erforderlichen Instrumente zur Zielerfüllung gar nicht besitzen.

\section{Psychologisches Moment}

Auch 20 Jahre nach Einführung des EEG kann die Politik - vielleicht: jetzt erst recht - nicht zugeben, dass die Energiewende zu scheitern droht. Die Politiker scheinen die hohen ökonomischen Kosten und Ineffizienzen des EEG zu ignorieren und die Verfehlung der Klimaschutzziele nur halbherzig zu bedauern. Und hat Bundeskanzlerin Angela Merkel nicht ihre eigene Klimapolitik im Sommer 2019 als „Pillepalle“ bezeichnet und ihr damit selbst ein grottenschlechtes Zeugnis ausgestellt und eine deutliche Besserung mit dem sogenannten „Klimapaket 2030“ versprochen? Warum kann sich die Klimapolitik dann nicht ehrlicherweise eingestehen, dass sie mit ihrem „Großprojekt Energiewende“ gescheitert ist (Altrogge, 2019)?

Der Umgang mit wirtschaftlichem und gesellschaftlichem Scheitern ist in Deutschland eher negativ besetzt. Nach einer repräsentativen Umfrage der Universität Hohenheim steht nur etwa die Hälfte der Befragten dem unternehmerischen Scheitern positiv oder überwiegend positiv gegenüber (Kuckertz et al., 2015, 11 ff.). International vergleichende Studien zeigen auch, dass die Angst vor einem geschäftlichen Misserfolg in Deutschland deutlich höher ist als z. B. in den USA. Und während es im wissenschaftlichen Bereich systemimmanent ist, aus Fehlern zu lernen und seine fachliche Position auch mal zu ändern, werden im politischen Bereich eindeutige und stets korrekte Antworten erwartet. Politische Fehler eingestehen zu müssen, bedeutet dann oftmals das politische Aus.

Im politischen Bereich ist das Scheitern in einem öffentlichen Amt ein Sonderfall: Es geschieht unter den Augen der Öffentlichkeit und wird von den Medien meist kritisch begleitet und kommentiert. Öffentlichkeit und Medien sind nach eigener persönlicher Erfahrung der ehemaligen Oberbürgermeisterin von Kiel, Susanne Gaschke, die im Oktober 2013 von ihrem Amt zurückgetreten ist, eher bösartig, ungerecht und besserwisserisch (Gaschke, 2018). Denn es liege ja in der Natur der journalistischen Arbeit, dass sie Ergebnisse im Nachhinein beurteilt, ohne selbst politische Verantwortung tragen und Konsequenzen fürchten zu müssen. Gaschke (2014, 216 ff.) beklagt besonders die Skandalisierung von einzelnen politischen Entscheidungen und die persönliche Diffamierung, die zu Vorverurteilungen und einer medialen Zerstörung von einzelnen Politikern führen kann (wie im Fall des damaligen Bundespräsidenten Christian Wulff). Es scheint unterschiedliche Toleranzniveaus für das Scheitern in verschiedenen gesellschaftlichen Bereichen zu geben. Wenn das politische Scheitern am wenigsten gesellschaftlich akzeptiert wird, so steht zu erwarten, dass im politischen Bereich am wenigsten - und schon gar nicht öffentlich eigene Fehler zugegeben werden können. Vielleicht wird auch damit verständlich, dass das Eingeständnis der Bundeskanzlerin über ihre Pillepalle-Klimapolitik nicht zu einer breiten gesellschaftlichen Diskussion über die unbefriedigende Klimapolitik geführt hat.

\section{Schlussbetrachtung}

Die Bilanz der bisherigen Klimapolitik in Deutschland ist ernüchternd: Die klimapolitischen Ziele werden weitgehend verfehlt, und die gesellschaftlichen Kosten erscheinen demgegenüber sehr hoch. Und Politiker zeigen - gerade in Corona-Zeiten - wieder eine stärkere Präferenz für planwirtschaftliche und staatsinterventionistische Politikelemente. Die Klimapolitik in Deutschland scheint einer Realitätsillusion zu unterliegen.

Die klimapolitische Herausforderung ist aber ohne eine rationale Klimapolitik nicht zu bewältigen. Grundbedingung dafür ist, dass Klimapolitik auch von der Politik in Deutschland als internationales Koordinationsproblem erkannt und angegangen wird. Deutschland sollte sich - viel stärker als bisher - für einen weltweit einheitlichen $\mathrm{CO}_{2}$-Preis einsetzen und dafür werben, ihn schrittweise zu institutionalisieren (z. B. in der Gruppe der G20-Staaten). Die EU-Ratspräsidentschaft im 2. Halbjahr 2020 könnte Deutschland nutzen, zunächst in der EU für mehr marktorientierten Klimaschutz zu werben.

Darüber hinaus sind mehr Sachlichkeit und Vernunft in Deutschland über die Instrumente der Klimapolitik dringend erforderlich. Zentrale Elemente dafür müssen sein: Wirksamkeit, Kosteneffizienz, Technologieoffenheit und internationale Arbeitsteilung sowie Wettbewerbsfähigkeit und gesellschaftliche Akzeptanz. Angesichts der Ineffektivität und Ineffizienz der bisherigen deutschen Klimapoli- 
tik darf es ein noch stärkeres „Weiter so mit denselben Instrumenten" nicht geben. Notwendig ist ein grundlegender Strategiewechsel der deutschen Klimapolitik, der nur mit der Einführung eines einheitlichen $\mathrm{CO}_{2}$-Preises über alle Sektoren hinweg erreicht werden kann. Nur so können die Produktivkräfte der Marktwirtschaft effektiv und effizient gezielt für den Klimaschutz eingesetzt werden. Deutschland sollte endlich aufhören, mit hohen Subventionen den Ausbau der erneuerbaren Energien zu fördern. Letztlich muss es Ziel der Klimapolitik in Deutschland sein, nachhaltig zu demonstrieren, dass sich Emissionsminderungen mit hohem und wachsendem Wohlstand und gesellschaftlicher Akzeptanz verbinden lassen.

\section{Literatur}

Altrogge, G. (2019), Das misslungene Großprojekt. Die deutsche Energiewende droht zu scheitern. Nur zugeben will es niemand. Für die Politik wäre es ein Offenbarungseid, Welt am Sonntag, 15. September, 34.

Bardt, H. und T. Schaefer (2018), Verteilungsprobleme und Ineffizienz in der Klimapolitik, IW-Kurzbericht, 1.

Bernau, P. (2020), Lasst die Preise in Ruhe!, Frankfurter Allgemeine Sonntagszeitung, 9. Feburar, 20.

BMU (Bundesministerium für Umwelt, Naturschutz und nukleare Sicherheit) (2019), Klimaschutzprogramm 2030 der Bundesregierung zur Umsetzung des Klimaplans 2050, https://www.bmu.de/fileadmin/Daten BMU/Download_PDF/Klimaschutz/klimaschutzprogramm_2030_ umsetzung_klimaschutzplan.pdf (26. Oktober 2019).

Bundesrechnungshof (2018), Bericht nach \$ 99 BHO über die Koordination und Steuerung zur Umsetzung der Energiewende durch das Bundesministerium für Wirtschaft und Energie, 28. September.

Bundesregierung (2019), Eckpunkte für das Klimaschutzprogramm 2030, https://www.bundesregierung.de/resource/blob/975232/1673502/7 68b67ba939c098c994b71c0b7d6e636/2019-09-20-klimaschutzprogramm-data.pdf?download=1 (21. Spetember 2019).

Cassel, S. (2017), Politische Ökonomie der Energiewende: Reformen des EEG im Interessengeflecht, in Thieme, H. J. und J. Haucap (Hrsg.): Ordnungsdefizite und Lösungsansätze, 37-58.

$\mathrm{CO}_{2}$-Abgabe (2019), Energiesteuern klima- \& sozialverträglich gestalten. Wirkungen und Verteilungseffekte des $\mathrm{CO}_{2}$-Abgabekonzepts auf Haushalte und Pendelnde.

Dubbers, D., J. Stachel und U. Uwer (2019), Energiewende: Warum sich Deutschland verrechnet, Münchner Merkur, 14. Oktober, 3.

Ehler, T. (2019), Deutsche Energiewende als „Rent-Seeking Paradise“?, Energiewirtschaftliche Tagesfragen, 69(4), 43-45.

Erlei, M., M. Leschke und D. Sauerland (2016), Institutionenökonomik, 3. Aufl.

Fell, H.-J. (2020), „Ein nicht mehr handhabbares bürokratisches Monster“, Interview, Bayerische Staatszeitung, 27. März, 19.

Festinger, L. (1978/2019), Theorie der kognitiven Dissonanz, 3. Aufl., Göttingen.

Frondel, M. (2017), Ineffektiv und ineffizient. Eine Bilanz der deutschen Klimapolitik. Gutachten im Auftrag der Friedrich-Naumann-Stiftung für die Freiheit.
Fuest, C. (2020), Die schleichende Verbreitung des Neodirigismus, Frankfurter Allgemeine Zeitung, 7. Februar.

Gaschke, S. (2014), Volles Risiko. Was es bedeutet, in die Politik zu gehen, Deutsche Verlags-Anstalt.

Gaschke, S. (2018), Lasst die Häme weg!, Politisches Feuilleton, 18. Oktober, https://www.deutschlandfunkkultur.de/wenn-politiker-scheitern-lasst-die-haeme-weg.1005.de.html?dram:article_id=430777 (3. April 2020)

Göbel, H. (2019), Der Markt als Verbündeter im Klimaschutz, Aus Politik und Zeitgeschichte, 69(47-48), 18. November, 26-29.

Kemfert, C. (2019), Energiewende - Mythen reloaded, https://www.capital.de/wirtschaft-politik/energiewende-mythen-reloaded (21. April 2020).

Köcher, R. (2020), Auch Deutschland kommt bald an seine Grenzen, Handelsblatt, 28. April, 6-7.

Kübler, K. (2020), Zu den Wirkungen des Klimaschutzprogramms 2030 - Eine picoökonomische Analyse, Energiewirtschaftliche Tagesfragen, $70(3), 36-41$.

Lindner, C. (2005), Politik und Politikberatung - Versuch einer Verständigung, in Jens, U. und H. Romahn (Hrsg.): Glanz und Elend der Politikberatung, 67-83.

Monopolkommission (2009), Strom und Gas: Energiemärkte im Spannungsfeld von Politik und Wettbewerb, Sondergutachten 54.

Nassehi, A. (2019), Denkfaule Demokratieverächter, Süddeutsche Zeitung, 2. August, 9

Richter, S. (2020), Deutsche Lebenslügen, Handelsblatt, 10.-12. Januar, 64.

SVR (Sachverständigenrat zur Begutachtung der gesamtwirtschaftlichen Entwicklung) (2019), Aufbruch zu einer neuen Klimapolitik, Sondergutachten des SVR, 12. Juli, https://www.sachverstaendigenrat-wirtschaft.de/sondergutachten-2019.html (14. Juli 2019).

Schnellenbach, J. (2020), Das Klimapaket der Bundesregierung: Dirigismus statt Preise, Wirtschaftswissenschaftliches Studium, 1, 37-39.

Speth, R. (2004), Politikberatung als Lobbying, in Dagger, S. et al. (Hrsg.): Politikberatung in Deutschland, 164-177.

Techert, H., J. Niehues und H. Bardt (2012), Ungleiche Belastung durch die Energiewende: Vor allem einkommensstarke Haushalte profitieren, Wirtschaftsdienst, 92(8), 507-512.

Weimann, J. (2016), Internationale Lösung durch nationale Politik. Eine Bewertung des Pariser Klimaabkommens, ifo-Schnelldienst, 69(3), 3-5.

Weimann, J. und F. Timme (2019), Die Entfremdung des Emissionshandels, Energiewirtschaftliche Tagesfragen, 69(5), 29-32.

Wetzel, D. (2020), Bei der Energiewende könnte die Corona-Krise auch etwas Gutes haben, Die Welt, 15. Mai.

Wissenschaftlicher Beirat beim BMWi (2004), Zur Förderung erneuerbarer Energien. Gutachten, Stand: Juli, https://www.bmwi.de/Redaktion/DE/Publikationen/Ministerium/Veroeffentlichung-Wissenschaftlicher-Beirat/wissenschaftlicher-beirat-erneuerbare-energien-534. html (26. Oktober 2019).

Wissenschaftlicher Beirat beim BMWi (2016), Die essentielle Rolle des $\mathrm{CO}_{2}$-Preises für eine effektive Klimapolitik, https://www.bmwi.de/ Redaktion/DE/Publikationen/Ministerium/Veroeffentlichung-Wissenschaftlicher-Beirat/wissenschaftlicher-beirat-rolle-co2-preis-fuerklimapolitik.pdf?__blob=publicationFile\&v=20 (26. Oktober 2019).

Wissenschaftlicher Beirat beim BMWi (2019), Energiepreise und effiziente Klimapolitik, Gutachten, Stand: 28. Juni, https://www.bmwi.de/ Redaktion/DE/Publikationen/Ministerium/Veroeffentlichung-Wissenschaftlicher-Beirat/gutachten-energiepreise-effiziente-klimapolitik. pdf?__blob=publicationFile\&v=10 (14. Juli 2019).

Title: Reality-IIlusion of the Climate Policy of Germany

Abstract: Climate protection is a huge global challenge and climate policy can be characterised as an international public good problem. The climate policy of Germany is limited, expensive and inefficient and does not contribute to the desired reduction of the $\mathrm{CO}_{2}$-emissions. But why? Why are we in Germany subject to an illusion of climate policy? There are multiple reasons: Politicians seem unwilling to sufficiently learn and appear unable to concede they are wrong. Politics get entangeld in distributional conflicts with EEG lobby groups and orchestrate their pretend efforts in public. Politicians tend more to dirigistic than to market-based policy instruments and ease the urge towards distributional interventions in the market. These findings will be analysed in the light of the New Institutional Economics. 